\title{
Oscillation-Based Connectivity Architecture Is Dominated by an Intrinsic Spatial Organization, Not Cognitive State or Frequency
}

\author{
(ㄱ) Parham Mostame ${ }^{1,2}$ and ${ }^{-S e p i d e h ~ S a d a g h i a n i ~}{ }^{1,2}$ \\ ${ }^{1}$ Psychology Department, University of Illinois at Urbana-Champaign, Urbana, Illinois, 61820, and ${ }^{2}$ Beckman Institute for Advanced Science and \\ Technology, University of Illinois at Urbana-Champaign, Urbana, Illinois, 61801
}

Functional connectivity of neural oscillations (oscillation-based FC) is thought to afford dynamic information exchange across task-relevant neural ensembles. Although oscillation-based FC is classically defined relative to a prestimulus baseline, giving rise to rapid, context-dependent changes in individual connections, studies of distributed spatial patterns show that oscillation-based FC is omnipresent, occurring even in the absence of explicit cognitive demands. Thus, the issue of whether oscillation-based FC is primarily shaped by cognitive state or is intrinsic in nature remains open. Accordingly, we sought to reconcile these observations by interrogating the ECoG recordings of 18 presurgical human patients ( 8 females) for state dependence of oscillation-based FC in five canonical frequency bands across an array of six task states. FC analysis of phase and amplitude coupling revealed a highly similar, largely state-invariant (i.e., intrinsic) spatial component across cognitive states. This spatial organization was shared across all frequency bands. Crucially, however, each band also exhibited temporally independent FC dynamics capable of supporting frequency-specific information exchange. In conclusion, the spatial organization of oscillation-based FC is largely stable over cognitive states (i.e., primarily intrinsic in nature) and shared across frequency bands. Together, our findings converge with previous observations of spatially invariant patterns of FC derived from extremely slow and aperiodic fluctuations in fMRI signals. Our observations indicate that "background" FC should be accounted for in conceptual frameworks of oscillation-based FC targeting task-related changes.

Key words: amplitude coupling; electrocorticography; functional connectivity; intrinsic; oscillations; phase coupling

Significance Statement

A fundamental property of neural activity is that it is periodic, enabling functional connectivity (FC) between distant regions through coupling of their oscillations. According to task-based studies, such oscillation-based FC is rapid and malleable to meet cognitive task demands. Studying distributed FC patterns instead of FC in a few individual connections, we found that oscillation-based FC is largely stable across various cognitive states and shares a common layout across oscillation frequencies. This stable spatial organization of FC in fast oscillatory brain signals parallels the known stability of fMRI-based intrinsic FC architecture. Despite the observed spatial state and frequency invariance, FC of individual connections was temporally independent between frequency bands, suggesting a putative mechanism for malleable frequency-specific FC to support cognitive tasks.

\section{Introduction}

Oscillation-based functional connectivity (FC) is thought to be essential for neuro-cognitive processing (Singer, 1999; Varela et

Received Aug. 15, 2020; revised 0ct. 10, 2020; accepted Nov. 3, 2020.

Author contributions: P.M. and S.S. designed research; P.M. and S.S. performed research; P.M. analyzed data; P.M. wrote the first draft of the paper; P.M. and S.S. edited the paper; P.M. and S.S. wrote the paper.

We thank Dr. Graham Huesmann for discussions of ECOG data quality; and Dr. Kai Miller and colleagues for generously sharing their data.

The authors declare no competing financial interests.

Correspondence should be addressed to Parham Mostame at mostame2@illinois.edu.

https://doi.org/10.1523/JNEUROSCI.2155-20.2020

Copyright $\odot 2021$ the authors al., 2001; Engel et al., 2013). Depending on the particular frequency band, such FC supports performance in the broadest range of cognitive and behavioral domains, from perception and motor output (Khanna and Carmena, 2015; VanRullen, 2016) to attention (S. Palva and Palva, 2007; Jensen et al., 2014; Sadaghiani and Kleinschmidt, 2016) and language processing (Rimmele et al., 2018). Oscillation-based FC leverages a fundamental property of electrophysiological activity, namely, its periodicity in characteristic frequency bands. This reliance on neural oscillations dissociates oscillation-based FC from FC measures derived from broadband and aperiodic signals (e.g., Pearson correlation) extensively used in fMRI (M. D. Fox and 
Raichle, 2007) as well as some neurophysiological studies (e.g., Chu et al., 2012). Oscillation-based FC can be conceptualized in terms of two major modes (Engel et al., 2013; Mostame and Sadaghiani, 2020): (1) phase coupling denoting synchronization of the phase of neural activity of distinct brain regions over multiple consecutive oscillation cycles (Lachaux et al., 1999; Nolte et al., 2004); and (2) amplitude coupling denoting synchronization of the magnitude of neural oscillations between regions (Brookes et al., 2011).

Oscillation-based phase and amplitude coupling have traditionally been evaluated in a connection-wise manner across a small set of brain regions (Singer, 1999; Uhlhaas et al., 2009). A more recent advance is to derive large-scale connectivity maps from EEG or MEG source space (e.g., Deligianni et al., 2014; Hipp and Siegel, 2015; Tewarie et al., 2016) and intracranial data (Kucyi et al., 2018; Betzel et al., 2019). This advance is grounded in the understanding that the comprehensive FC organization is of functional importance, in part reflecting prior knowledge stored in the connectome (Sadaghiani and Kleinschmidt, 2013; Singer, 2013).

Such investigations of distributed patterns of oscillationsbased FC are commonly applied to the task-free resting state. At rest, brain oscillations persist to an overall similar extent than during explicit tasks (although their specific spectral distribution is modulated by cognitive demands). In the following, we collectively refer to task-free wakefulness and engagement in different behavioral tasks as cognitive states. For a century, task-independent ongoing oscillations have been the hallmark of EEG (Berger, 1930). The spectral profile of this ongoing activity and its cross-areal connectivity have proven informative for understanding brain function and dysfunction (de Medeiros Kanda et al., 2009; Sadaghiani et al., 2019). Source-localized MEG and EEG studies have reported the presence of a distributed spatial organization or "architecture" of oscillation-based FC at resting state (Hillebrand et al., 2012; Colclough et al., 2016; Sockeel et al., 2016; Wirsich et al., 2017). Intracranial cortical surface recordings of presurgical patients (electrocorticography $[\mathrm{ECoG}]$ ) have confirmed this architecture (K. C. Fox et al., 2018; Kucyi et al., 2018; Betzel et al., 2019) in the absence of the ill-posed problem of EEG/MEG source reconstruction (Schoffelen and Gross, 2009; J. M. Palva et al., 2018). However, these studies have not quantitatively assessed (dis)similarity of oscillation-based FC organization across different cognitive states. Instead, contrasts across task conditions or between poststimulus and prestimulus intervals are typically investigated in a connection-wise manner. Therefore, the degree to which large-scale FC organization of oscillatory rhythms is modulated by cognitive context is largely unknown.

To summarize, electrophysiological oscillations and their FC express strong context-dependent flexibility on the one hand and are omnipresent across all cognitive states on the other. This tension therefore begs the question of whether oscillation-based FC is largely governed by a state-invariant (i.e., intrinsic) spatial organization or is primarily dependent on cognitive demands.

A few neurophysiological studies have taken important steps toward answering this question. Kramer et al. (2011) analyzed daylong ECoG recordings across various levels of arousal and states of consciousness. Static FC across electrodes during periods $>100$ s displayed consistent spatial organization over the course of the day. However, this study did not directly and quantitatively contrast FC across the different consciousness levels. A subsequent scalp EEG study identified high spatial correlation of static (>100 s) FC organization in sensor space over different sleep stages and wakefulness $(r>0.75)$ (Chu et al., 2012). However, different cognitive activities were not dissociated during the waking period. It is therefore unclear how these findings relate to trial-based oscillatory FC commonly investigated in cognitive neuroscience. A more recent scalp EEG study showed that phase coupling in reconstructed source space is consistent across tasks (resting state, video viewing, and flashing gratings), with FC clusters that are reproducible across frequency bands (Nentwich et al., 2020). However, while promising, the latter two studies must be interpreted with care because of the methodological limitations imposed by EEG recorded over the scalp, which may lead to spurious FC, even in source space (J. M. Palva et al., 2018). Together, these findings motivate direct comparison of static FC organization across cognitive states using high fidelity intracranial data.

Dependence on cognitive context has been well quantified in another research field, namely, the study of very slow FC derived from aperiodic BOLD fluctuations observed with fMRI. The brain at resting state displays covariation in BOLD signals across specific sets of regions (i.e., intrinsic connectivity networks) (Beckmann et al., 2005). Importantly, this intrinsic functional architecture is also present during task, suggesting that task-specific changes to the brain's fMRI-derived FC spatial organization are small (Smith et al., 2009; Cole et al., 2014; Hearne et al., 2017; Gratton et al., 2018). For example, Cole et al. (2014) identified a strong spatial correlation $(r=\sim 0.9)$ between the static FC organization of rest and task data.

However, whether these fMRI-based findings can inform about potential state invariance in oscillation-based FC is questionable, given that $\mathrm{fMRI}$-derived FC is an indirect measure of neural activity based on aperiodic fluctuations of the BOLD signal in the infra-slow range (mainly $<0.01 \mathrm{~Hz}$ ) (M. D. Fox and Raichle, 2007; Thompson and Fransson, 2015). In contrast, FC as measured by electrophysiological methods reflects real-time neural processes driven by cyclic activity. As such, oscillationbased FC is well positioned to support long-range communication required for cognitive processes that unfold on the rapid timescale of tens to hundreds of milliseconds (Uhlhaas et al., 2009; Fell and Axmacher, 2011; Gruber et al., 2018). Given these fundamentally different characteristics of the processes associated with fMRI-based and oscillation-based FC, the degree of context dependence of the latter requires dedicated investigation.

Another characteristic of fundamental functional importance unique to oscillation-based FC is its frequency dependence. In particular, within the context of different tasks specific, cognitive functions are associated with oscillatory processes in distinct frequencies (e.g., S. Palva and Palva, 2007; Rohenkohl et al., 2018). Based on this functional specificity of particular oscillation bands and their association with different brain regions, should we expect the oscillation-based FC organization in different frequency bands to differ from each other? This question remains largely unanswered because task-based studies traditionally focus on task-related modulations in individual connections, ignoring the distributed spatial organization of oscillation-based FC. The observation of a spatial organization shared across frequency bands has implications for the neurobiological understanding of functional networks. Specifically, such frequency invariance, if observed, would suggest that large-scale functional networks may enact connectivity in all canonical frequencies regardless of the sensory, motor, or cognitive operation that they subserve and the regions that they connect.

In light of the foregoing, we addressed two central questions in the current study: (1) Is the spatial organization of fast 


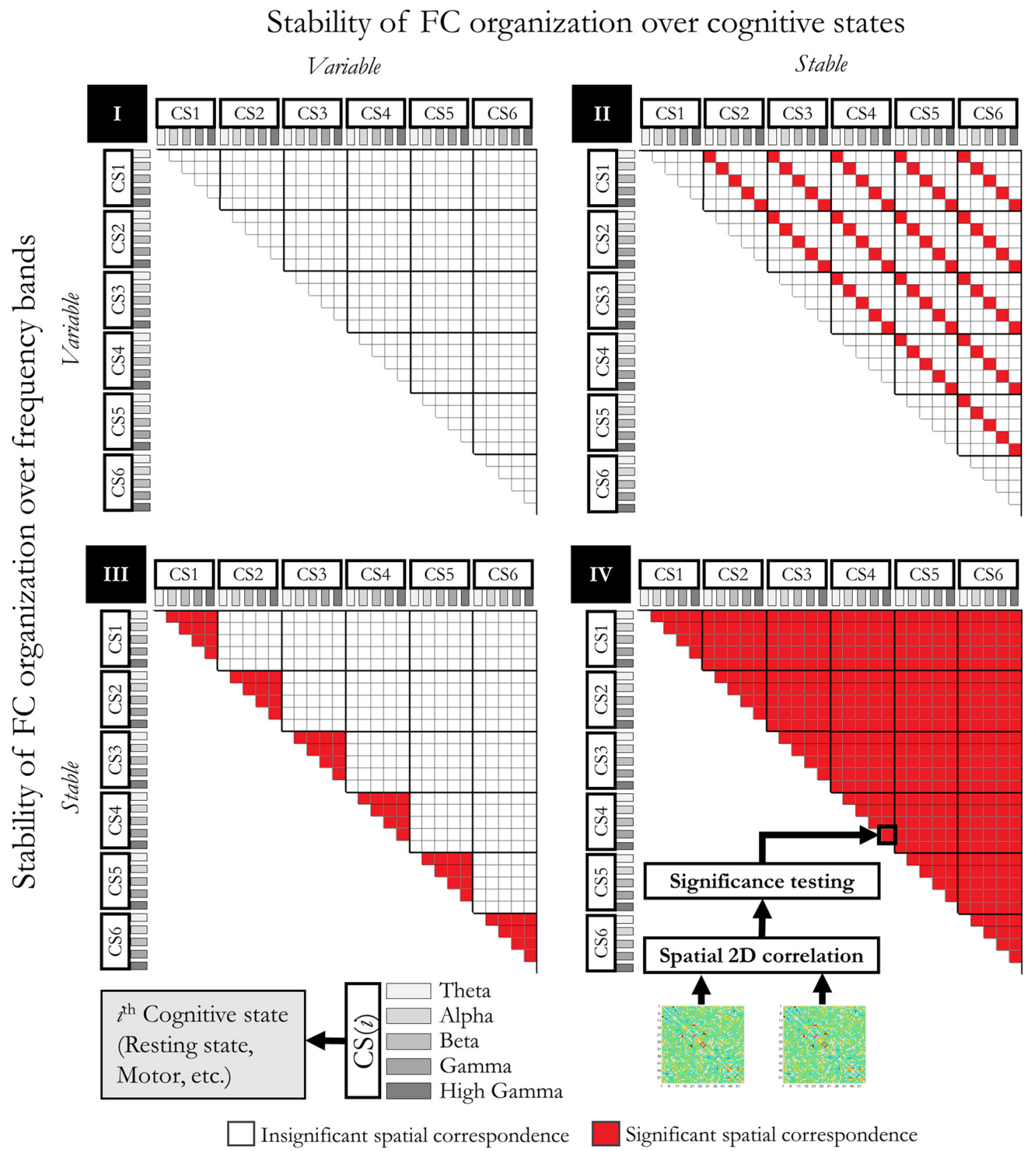

Figure 1. Four hypothetical scenarios (I-IV) regarding stability of the spatial organization of FC across cognitive states and frequency bands. Each cognitive state CSi is subdivided into five frequency bands (theta, $\alpha, \beta, \gamma$, and high $\gamma$ ). Each element within the matrix represents the $2 \mathrm{D}$ spatial correspondence (quantified as correlation) between corresponding pairs of $\mathrm{FC}$ matrices, where red represents a statistically significant relationship. Our results will be explicitly compared with these four scenarios in Figure 6 .

oscillation-based FC primarily driven by cognitive operations or is it stable across task states? (2) Is this organization dependent on oscillation frequency? Following these two central investigations, we further asked: (3) Does frequency-invariant FC organization (if observed) reflect a single broadband coupling process or truly multiple temporally independent and frequency-specific processes within a universal spatial organization? Finally: (4) Does the hypothesized stability of static FC organization over cognitive states and frequency bands depend on the specific connectivity mode (i.e., phase or amplitude coupling)? To address these questions while minimizing the impact of volume conduction on FC (Schoffelen and Gross, 2009; J. M. Palva et al., 2018), we study ECoG in patients undergoing clinical evaluation for epilepsy surgery. We further replicate major findings using additional FC measures that suppress the impact of potential volume conduction. In summary, we dissociate among four possible scenarios of state and frequency dependency of FC organization as illustrated in Figure 1.

\section{Materials and Methods}

Data

In this study, we used publicly available ECoG recordings during resting state and three independent paradigms comprising a total of six distinct cognitive states. The data are described in detail by Miller (2019) and are available at https://searchworks.stanford.edu/view/zk881ps0522. All patients participated in a purely voluntary manner, after providing informed written consent, under experimental protocols approved by the Institutional Review Board of the University of Washington (\#12193). All patient data were anonymized according to Institutional Review Board protocol, in accordance with the Health Insurance Portability and Accountability Act mandate. 
Subjects. From the above-mentioned library, we included only those subjects who had data in at least two paradigms in such a way that maximized the number of subjects for pairs of cognitive states (see below). Cumulatively over the chosen task pairs, the dataset contained 18 human subjects ( 8 females, 7 males, and 3 subjects without reported sex information). However, not all subjects had data in all of the six cognitive states (median number of states across subjects $=3$ ). Subjects, disregarding three with missing age information, were on average 30.8 years old (SD 9.3 years). The average number of electrodes per subject was 53.5 (SD 12.7) with an interelectrode distance of $1 \mathrm{~cm}$. Figure 2 shows the electrode location maps of all subjects. As shown in Figure 2, all subjects have electrodes over at least two of the frontal, temporal, and parietal lobes in either the left or right hemisphere, suggesting coverage of multiple functionally distinct regions. All data were sampled at $1000 \mathrm{~Hz}$, with a built-in $0.15-200 \mathrm{~Hz}$ bandpass filter. Beyond the initial data cleaning performed by Miller (2019), including rejection of channels with obvious artifact or epileptic activity, portions of the data with excessive interictal activity were removed if necessary.

\section{Experimental design and statistical analysis}

Behavioral paradigms. In order to increase the statistical power of cross-state comparisons of FC, we used those paradigms that had the highest number of subjects with highest possible number of independent cognitive states: resting state, cue-based motor task, verb generation task, and 2-back task datasets. These tasks represent a highly diverse set of cognitive states in terms of cognitive demands. Motor and speech tasks have a prestimulus interval, whereas the 2-back task uses a continuous block design. As a result, we investigated the intrinsic FC across six cognitive states, including the following: resting state (Rest), prestimulus motor $\left(\right.$ Base $_{\text {Motor }}$ ), prestimulus speech (Base Speech $_{\text {) }}$, poststimulus motor (Motor), poststimulus speech (Speech), and 2-back.

Resting state (Rest): All subjects underwent restingstate recordings while fixating on an $\mathrm{X}$ on the wall $3 \mathrm{~m}$ away, for 2-3 $\mathrm{min}$ (for more information regarding these data, see Miller et al., 2009, 2012).

Cue-based motor task (Motor): Seventeen of the 18 subjects had motor task data. Patients were instructed to repetitively flex and extend all fingers based on a visual word cue indicating the body part that should be moved (an alternative cue in a separate run instructed tongue movements not analyzed in this study). Movements were self-paced with a rate of $\sim 1-2 \mathrm{~Hz}$ using the hand contralateral to the side of the cortical grid placement. Each movement interval was 3 -s-long, preceded by a rest interval of the same length (blank screen). To maximize independence between trials, we excluded $0.5 \mathrm{~s}$ from the two tails of the trials, resulting in a $[-2.5,2.5 \mathrm{~s}]$ interval relative to cue onset. We analyzed two task states comprising the prestimulus $([-2.5,0 \mathrm{~s}])$ and poststimulus $([0,2.5 \mathrm{~s}])$ intervals. There were between 30 and 75 trials of rest and movement per subject. For more detailed information of this task, see Miller et al. (2007). Miller et al. (2007) have reported robust task-related power changes in the high-frequency or high $\gamma$ range in all subjects, implying that the subjects have task-appropriate electrode coverage suitable for our study.

Verb generation task (Speech): Five of the 18 subjects had performed the speech task. The speech data of 1 subject were excluded because of mismatching electrode grids with respect to the other tasks. Written nouns $(\sim 2.5 \mathrm{~cm}$ high and $8-12 \mathrm{~cm}$ wide) were presented on a screen positioned $\sim 1 \mathrm{~m}$ from the patient, at the bedside. Patients were asked to speak a verb that was semantically related to the noun. For example, if the noun read "ball," the patient might say "kick"; or if the noun read "bee," the patient might say "fly." Between each 1.6 s noun, there was a blank screen $1.6 \mathrm{~s}$ interstimulus interval. To avoid overlap between the
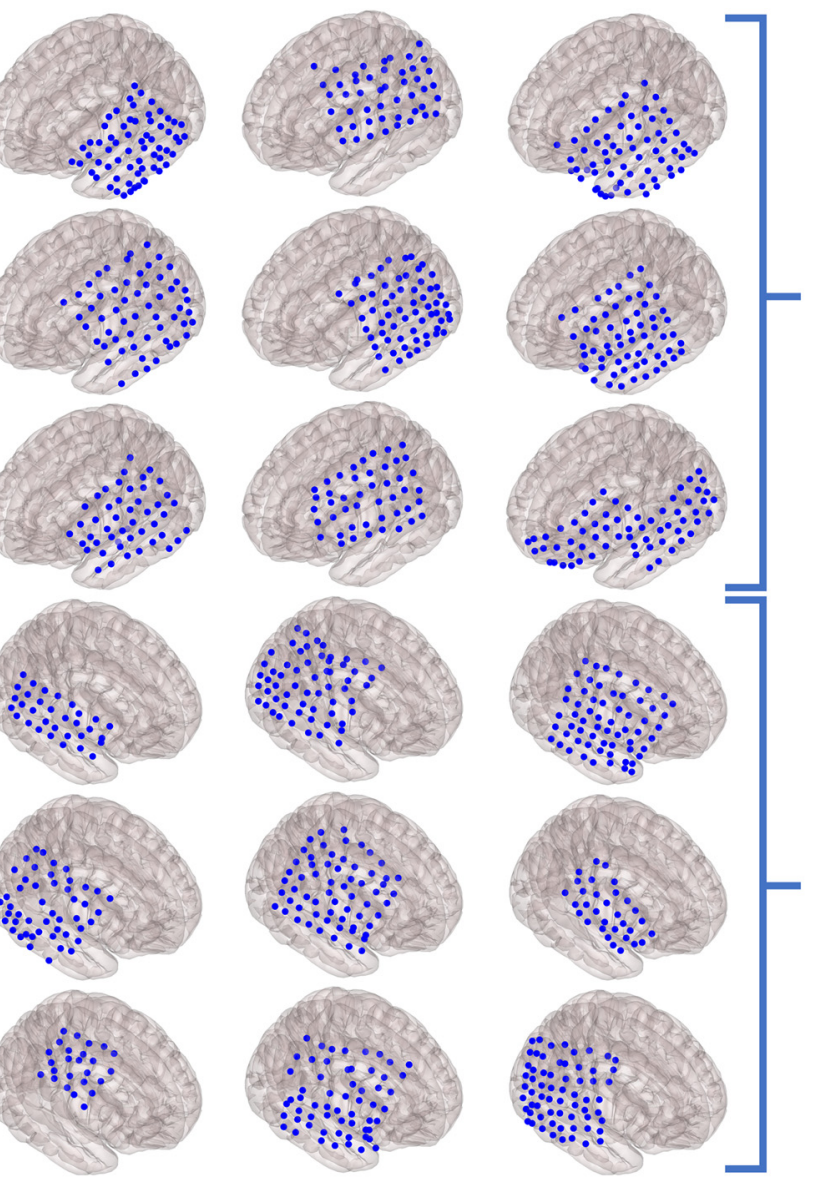

\section{草}



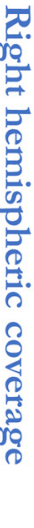

Figure 2. Electrode location maps of all subjects. Only electrodes that were clear of obvious artifacts and epiic activity (compare Miller, 2019) and were shared across conditions (e.g., Rest and Motor) are shown here 列 , whereas the other 9 subjects have right hemispheric electrode coverage. In each subject, at least two of the frontal, temporal, and parietal lobes are covered by the electrode grids.

trials during data analysis, we defined the trials from -1.5 to $1.5 \mathrm{~s}$ with respect to stimulus onset. We analyzed two task states comprising the prestimulus $([-1.5,0 \mathrm{~s}])$ and poststimulus $([0,1.5 \mathrm{~s}])$ intervals. Further details of the speech task data are provided in a prior investigation using these data (Miller et al., 2011).

2-back task (2-back): Four subjects of the 18 had performed the 2-back task. Among the available task conditions (0-back, 1-back, and 2back), we used 2-back since it requires higher cognitive involvement (e.g., attention and decision-making), which warrants larger possible divergence of cognitive state from resting state and the simple motor task. The stimuli comprised pictures of 50 houses, 10 of which were 2-back targets, each presented for $600 \mathrm{~ms}$. Subjects were instructed to flex their finger when a picture was the same as the one presented two trials earlier (i.e., target) (for more details, see Miller, 2019). Because the Nback task consists of a continuous stream of pictures, we considered task engagement to be continuous. Thus, in the FC estimations of 2-back, we analyze these data exactly same as the resting-state data after concatenating all respective blocks (see Static functional connectivity).

Data preprocessing. The publicly available data were initially cleaned (Miller, 2019). However, visual inspection of the signal time courses indicated the need for further cleaning in 2 subjects. Brief periods of epileptiform activity were thus manually marked and removed from the data of those subjects. Line noise was removed using two $60 \mathrm{~Hz}$ and $120 \mathrm{~Hz}$ second-order Butterworth notch filters. To remove low-frequency drift and high-frequency noise, data were filtered by low-pass $(120 \mathrm{~Hz}$ cutoff) and high-pass $(2 \mathrm{~Hz})$ fourth-order Butterworth filters. For task data, stimulus-locked trials were based on the prestimulus and poststimulus intervals described above. All data analyses were 
implemented in MATLAB, using FieldTrip (Oostenveld et al., 2011) and custom codes that can be found here: https:/github.com/connectlab/ IntrinsicFC_Mostame.

Our results primarily focus on phase coupling. Corresponding results for amplitude coupling are also provided in a more compact fashion in the corresponding sections. Phase and amplitude coupling are directly compared in Stability of spatial organization across phase and amplitude coupling (see Fig. 6). We estimated FC across five canonical frequency bands, including the following: theta $(5-7 \mathrm{~Hz}), \alpha(8-13 \mathrm{~Hz}), \beta(14-30 \mathrm{~Hz}), \gamma(31-$ $60 \mathrm{~Hz})$, and high $\gamma(61-110 \mathrm{~Hz})$. The $\delta$ band $(\sim 1-4 \mathrm{~Hz})$ was not included since the relatively short trial periods did not permit its reliable estimation from few oscillation cycles. We note that the use of canonical bands offers only a rough delineation of oscillatory processes, and separating the latter from broadband processes and defining them on an individual basis may provide refined results in the future. Below, static and dynamic estimation of phase and amplitude coupling is described.

Static functional connectivity. Static FC was estimated in a single time window for prestimulus $\left(\right.$ Base $_{\text {Motor }}$ and Base Speech $\left._{\text {n }}\right)$ and poststimulus intervals (Motor and Speech). Static FC was assessed in terms of phase coupling using the phase locking value (PLV) (Lachaux et al., 1999) as follows:

$$
P L V(f)=\frac{1}{N}\left|\sum_{n=1}^{N} e^{j \Delta \varphi_{i}(f)}\right|
$$

where $f$ is frequency, $N$ is number of trials, and $\Delta \varphi_{n}$ is the phase difference between the corresponding frequency components of the two electrode signals in trial $n$.

To derive amplitude coupling, the envelopes of pairwise band-limited signals (estimated through Hilbert transform) were correlated. Subsequently, temporal correlation values of all trials were averaged to obtain a single value for each electrode pair (ranging from -1 to 1 ), over the corresponding frequency band. To facilitate comparison with PLV, which ranges from 0 to -1 , absolute values of amplitude coupling were used as follows:

$$
\text { Amplitude_coupling }(f)=\frac{1}{N}\left|\sum_{n=1}^{N} \operatorname{corr}\left(\operatorname{env}\left(x_{1, n}^{f}\right), \operatorname{env}\left(x_{2, n}^{f}\right)\right)\right|
$$

Where $f$ is frequency, $N$ is number of trials, $\operatorname{corr}()$ is Pearson correlation, $e n v()$ is signal envelope driven from Hilbert analysis, and $x_{i, n}^{f}$ is frequency-specific component of $i^{\text {th }}$ electrode signal $(i=1,2)$ on the $n^{\text {th }}$ trial.

Static FC for the continuous conditions Rest and 2-back was estimated by averaging the dynamic FC (as defined below) over time.

Dynamic functional connectivity. Dynamic FC was used to dissociate broadband versus independent band-limited contributions to the dynamics of the intrinsic FC architecture. Windows were shifted every 1 $\mathrm{s}$, and window length varied as a function of canonical frequency band $(75,100,200,400$, and 800 cycles for theta, $\alpha, \beta, \gamma$, and high $\gamma$ frequency bands, respectively). Dynamic amplitude coupling was estimated as described in the static framework for each time window, when $N$ is equal to 1 . However, dynamic phase coupling was estimated using an alternate method.

In contrast to task-based phase coupling, commonly estimated as phase-lag consistency over trials (Lachaux et al., 1999), continuous phase coupling was estimated as phase-lag consistency over time (Sadaghiani et al., 2012; Mostame and Sadaghiani, 2020). The above-defined PLV measure adjusted to continuous data are defined as follows:

$$
P L V_{\text {Rest }}(f)=\frac{1}{M}\left|\sum_{m=1}^{M} e^{j \Delta \varphi_{m}(f)}\right|
$$

Where $M$ is the number of time samples within the time window.

Presence of intrinsic architecture. To assess whether FC organization is stable across cognitive states (Fig. 1; Scenarios II, IV vs Scenarios I, III), spatial Pearson correlations were calculated for each state pair. Cross-state correlations were tested against a null model that spatially permuted one of the matrices 500 times $(q<0.05$; Benjamini-Hochberg
FDR-corrected for number of subjects $\times$ frequencies $\times$ task states). Specifically, the phase of the 2D Fourier transform of the matrix was shuffled while keeping the amplitude intact. The odd symmetry of phases was preserved over frequencies in the $2 \mathrm{D}$ domain. The matrix was reconstructed using the inverse 2D Fourier transform, and its spatial correlation with the other matrix (which was not shuffled) assessed (Prichard and Theiler, 1994; Tewarie et al., 2016; Wirsich et al., 2017).

To determine the effect size of cross-state correlations, spatial stability of FC within Rest was estimated for comparison. Rest data of each subject were divided into two parts of equal length, and the similarity (2D Pearson correlation) between the static matrices of the two parts was calculated (see Fig. 3)

Shared intrinsic architecture across frequency bands. To assess whether FC in different oscillation frequencies share a similar intrinsic architecture (Fig. 1; Scenarios III, IV vs Scenarios I, II), spatial similarity between the static FC organization of all pairs of frequency bands was calculated. Since the intrinsic architecture is by definition considered stable across cognitive states (Cole et al., 2014; Petersen and Sporns, 2015), we estimated such an intrinsic organization for each frequency band by taking the geometric mean of its static FC organization over all cognitive states (Rest, Base Motor Base $_{\text {Speech }}$, Motor, Speech, and 2-back). Then, we calculated spatial correlation of this "frequency-specific" intrinsic architecture over every pair of frequency bands (see Fig. 4).

Broadband versus band-limited coupling events. Spatial correlation of FC organization across frequency bands may be driven by multiple band-limited processes with a similar spatial organization or, alternatively, a single broadband process. To dissociate between these scenarios, we compared FC time courses of different frequency bands. The procedure was applied only to the resting-state data because of limited length of trial-based task data. To focus on the frequency-invariant connections, only the top $25 \%$ strongest connections among all cross-state geometrical mean FC matrices of all bands were considered (mean connectivity strength $\pm \mathrm{SD}=0.60 \pm 0.13$ pooled over all connections and frequency bands and averaged over subjects). Within the selected connections, temporal correlations between phase coupling time courses (see Dynamic functional connectivity) were estimated over pairs of different bands. Finally, for each subject and frequency pair, all temporal correlations were pooled over connections (shown in Fig. 5).

To test to what degree the FC dynamics of two bands are systematically correlated, we generated a set of 500 surrogate data by phase-permuting the FC time course of one of the frequency bands (for each frequency pair and subject). For each permutation, we extracted the histogram of temporal correlations across all electrode pairs between the original and phase-permuted FC dynamics. The 500 surrogate histograms were pooled into a single histogram for each subject and frequency pair (see Fig. 5). To assess whether the original histogram was different from the surrogate histogram (indicating systematic correlation between frequency bands in the original data), we compared their mean, $\mathrm{SD}$, and skewness $(q<0.05$; FDR-corrected for all subjects, pairs of frequency bands, and three histogram measures).

Shared intrinsic architecture across connectivity measures. To directly assess the spatial similarity of intrinsic FC organization across phase and amplitude coupling as well as over all frequencies and cognitive states, we estimated spatial Pearson correlation between all possible pairs of FC matrices (across FC measures, cognitive states, and frequency). Finally, we averaged the resulting value of each pairwise comparison across all subjects (see Fig. 6, upper diagonal). We tested the significance of each bin by using the same $2 \mathrm{D}$ permutation method described above, phase-permuting one of the two matrices 500 times ( $q<0.05$; FDR-corrected by Benjamini-Hochberg method).

Addressing source leakage. Source leakage refers to the simultaneous detection of a particular brain signal (as a source) at several sensors because of volume conduction (Schoffelen and Gross, 2009), which may generate spurious connectivity between electrode pairs, especially in neurophysiological scalp recordings, such as EEG and MEG (J. M. Palva et al., 2018). Although much less of a concern for ECoG, we tested whether our results are affected by this potential confound.

First, following equivalent procedures for task and rest conditions as described for PLV, we used imaginary part of coherency (ImC) introduced 


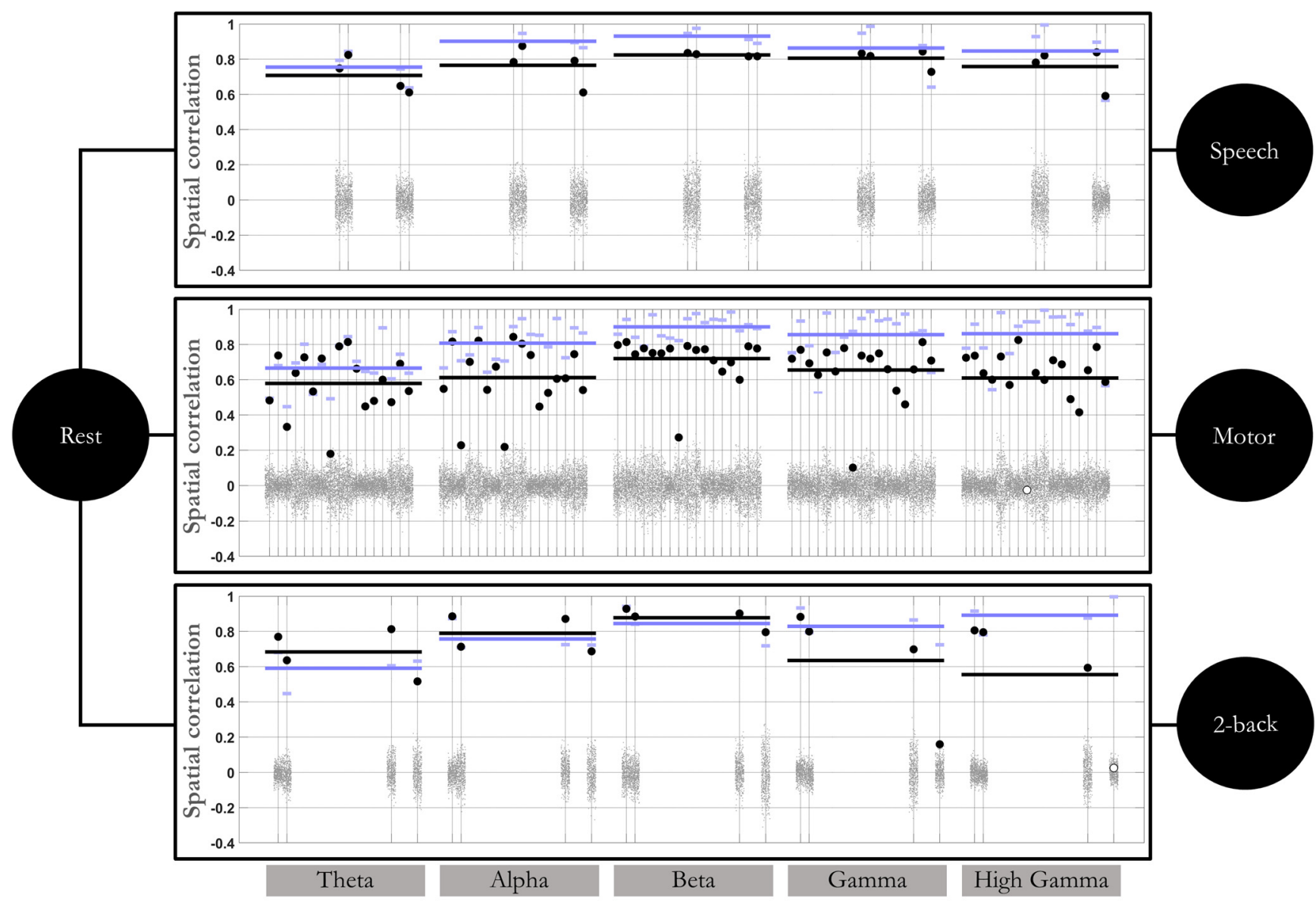

Single-subject cross-state spatial correspondence

Single-subject within-rest spatial correspondence
Average of cross-state spatial correspondence

Average of within-rest spatial correspondence
Null distribution for cross-state spatial correspondence

Figure 3. Example of spatial correspondence between $\mathrm{FC}$ matrices over three pairs of rest versus task states. States include task-free resting-state (Rest), verb generation (Speech), cue-based finger flexing (Motor), and memory recall (2-back). Not all subjects had data in all these states. In each subplot, frequency band is on the $x$ axis and spatial correlation on the $y$ axis. In each column of the plots, black dots represent the cross-state correlation of each subject. Long horizontal black line indicates average across subjects. Short horizontal purple lines indicate the within resting-state correlations of $\mathrm{FC}$ organization in each subject. Long horizontal purple line indicates average across subjects. The order of subjects is preserved across subplots to allow subject-specific comparison between cross-state pairs. Gray dot clouds represent the null model for the corresponding subject, frequency band, and cross-state pairing. The null model was generated by randomly permuting one of the matrices in 2D Fourier space. Pairwise correlations significantly exceed the null model in the vast majority of individual subjects and frequency bands (2 exceptions of the 125 pairs shown here), supporting the presence of a stable intrinsic FC organization.

by Nolte et al. (2004). ImC is the most commonly used measure suppressing zero-lag connectivity. This common approach rests on the assumption that electricity spreads quasi-instantaneously but comes at the cost of also removing real zero-lag connectivity (M. X. Cohen, 2015). Therefore, as a second approach, we regressed out electrode distance dependencies from FC values. Given the fact that volume conduction is dependent on electrode distance (Dubey and Ray, 2019; Rogers et al., 2019; Rouse et al., 2016), this approach is expected to substantially dampen possible volume conduction effects. Specifically, we fit cubic spline curves to the mean of the FC values within each nonoverlapping $1 \mathrm{~cm}$ range of electrode distance. We subtracted the value of the fitted curve from all corresponding FC values. This procedure removes any collinearity between the two measures resulting from their dependence on electrode distance. Once zero-lag FC or distance dependencies were removed, we estimated the cross-state spatial correlations as in Source leakage contributions (see Fig. 7).

\section{Results}

FC matrices were extracted for all subjects, cognitive states, and canonical frequency bands. First, to establish the presence of a cognitive state-invariant (i.e. intrinsic), FC organization, spatial correlation of the FC matrix across Rest and the three active task processing states (e.g. Motor) were assessed within each frequency band. Subsequently, the intrinsic FC organization most representative of each frequency was defined as the (geometric) mean of FC matrices across all six cognitive states in that frequency. Next, this representative organization was spatially compared across all bands to quantify frequency invariance of FC organization. Then, we compared FC time courses across frequency bands to address whether the spatial frequency invariance indeed reflects band-limited coupling in temporally independent frequencies or, alternatively, a broadband coupling phenomenon. Finally, to identify the most likely scenario from the four hypothetical scenarios illustrated in Figure 1, the spatial similarity across all pairs of FC matrices for all cognitive states and frequency bands was assessed.

\section{A state-invariant intrinsic FC organization}

Cross-state spatial correspondence in real versus surrogate data Spatial correlations between FC matrices of Rest and the three active task processing states were assessed in every frequency band (Fig. 3, black circles). All three pairs of cognitive states demonstrated strong spatial similarity in all frequencies (average $r=0.67 ; \mathrm{SD}=0.18$ ). Spatial correlations were compared with 
A

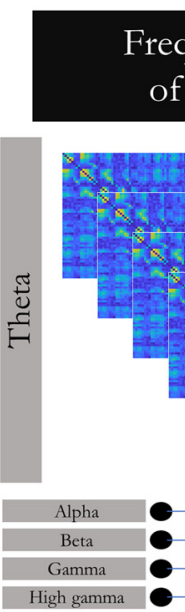
Rest theta-FC
B

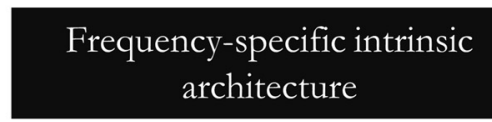

C

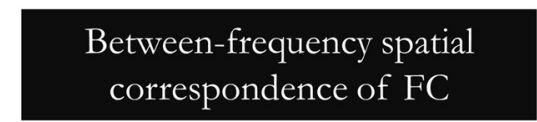

f all mental states

Baseline $_{\text {rotos }}$ theta-FC

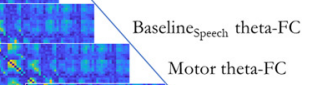

Motor theta-FC

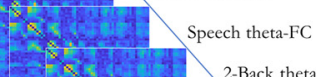

2-Back theta-FC
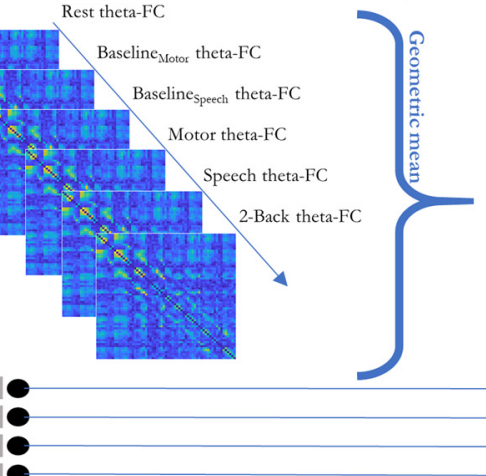

Theta band intrinsic architecture

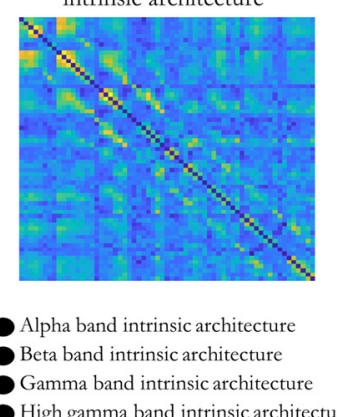

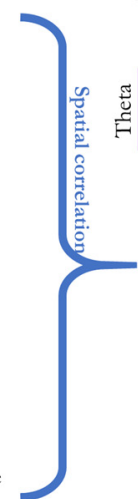

High

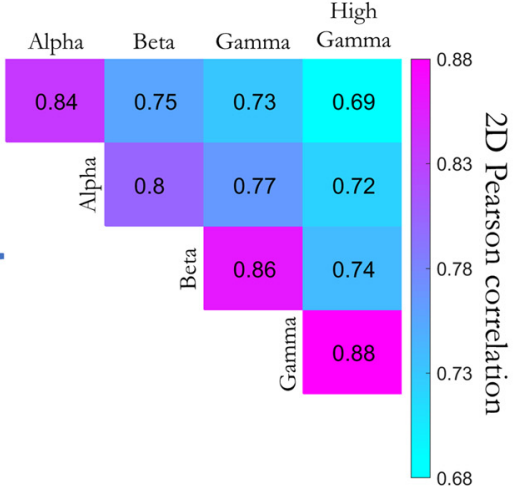

Figure 4. Spatial similarity of intrinsic $F C$ organization across frequency bands $(\boldsymbol{A}, \boldsymbol{B})$. $\boldsymbol{A}$, Schematic example of $\mathrm{FC}$ matrices from all cognitive states available for a given subject shown for the theta band. $B$, State-invariant representation of intrinsic $\mathrm{FC}$ organization of theta band was calculated by taking the geometric mean of the theta band $\mathrm{FC}$ matrices shown in $\boldsymbol{A}$. For each subject, such frequency-specific intrinsic architecture was separately extracted for each frequency. The ensuing frequency-specific intrinsic organization entered spatial correlation analysis across pairs of frequency bands. $C$, The between-frequency spatial correlation values averaged across all subjects. Rows/columns indicate frequency bands from theta to high $\gamma$. Correlation values of all frequency pairs were statistically significant across all individual subjects. The high correlation values $(r>0.69)$ indicate the presence of an intrinsic organization that is spatially similar across frequency bands.

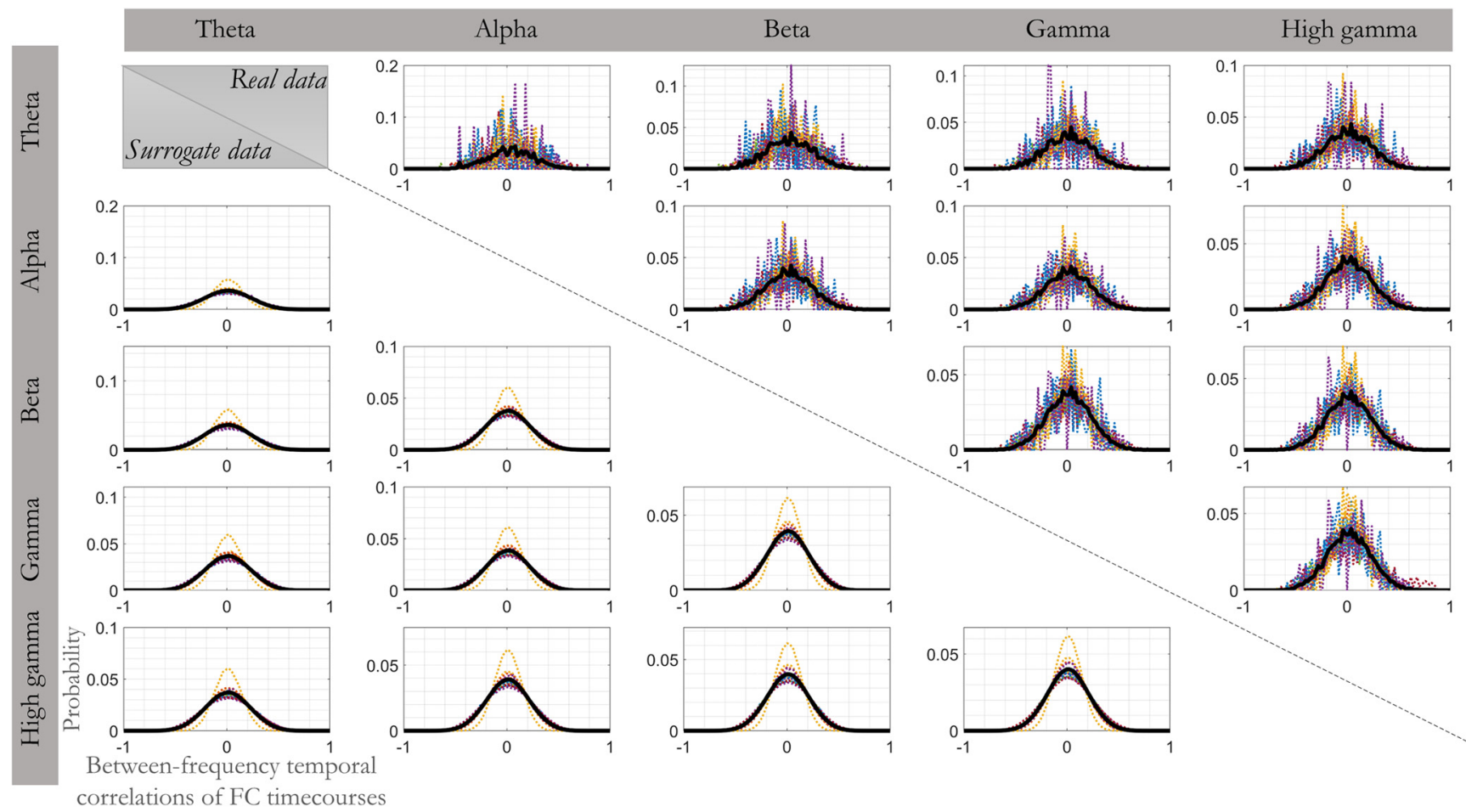

Figure 5. Histogram of temporal correlation values between resting-state $\mathrm{FC}$ dynamics of different frequency bands for real (top diagonal) and surrogate (bottom diagonal; pooled over 500 repetitions) data. The surrogate data represent temporally phase permuted and thus temporally independent $\mathrm{FC}$ time courses. Each panel corresponds to a specific pair of frequency band as labeled. In each panel, histograms averaged over subjects are shown as thick black lines, while single-subject histograms are shown as narrow dotted lines of different colors. $x$ axis indicates temporal Pearson correlation values of FC dynamics of the two frequency bands. $y$ axis indicates the probability of observing the respective values over electrode pairs. The histograms for real data were zero-centered and symmetric, as were those observed for the simulated temporally independent $\mathrm{FC}$ dynamics. This observation is consistent with the presence of multiple frequencyspecific processes with temporally independent time-varying dynamics rather than a single broadband phenomenon.

surrogate correlations generated after spatially shuffling one of the two matrices in 2D Fourier space (500 permutations; Fig. 3, gray dot clouds). At the group level, we compared the subjectspecific cross-state correlations (averaged over all cross-state conditions) with the mean value of the corresponding null models (averaged over permutations and all cross-state conditions).
For all frequency bands, cross-state spatial similarity exceeded the respective null model (paired $t$ test significant at $q<0.05$ with FDR correction, listed for theta through high $\gamma$ in ascending frequency: $t_{(17)}=3.90, p<0.005 ; t_{(17)}=3.86, p<0.005$; $t_{(17)}=5.81, p<0.005 ; t_{(17)}=3.06, p<0.005 ; t_{(17)}=2.45$, $p<0.05)$. At the individual level, the spatial similarity across 


\section{Phase Coupling}

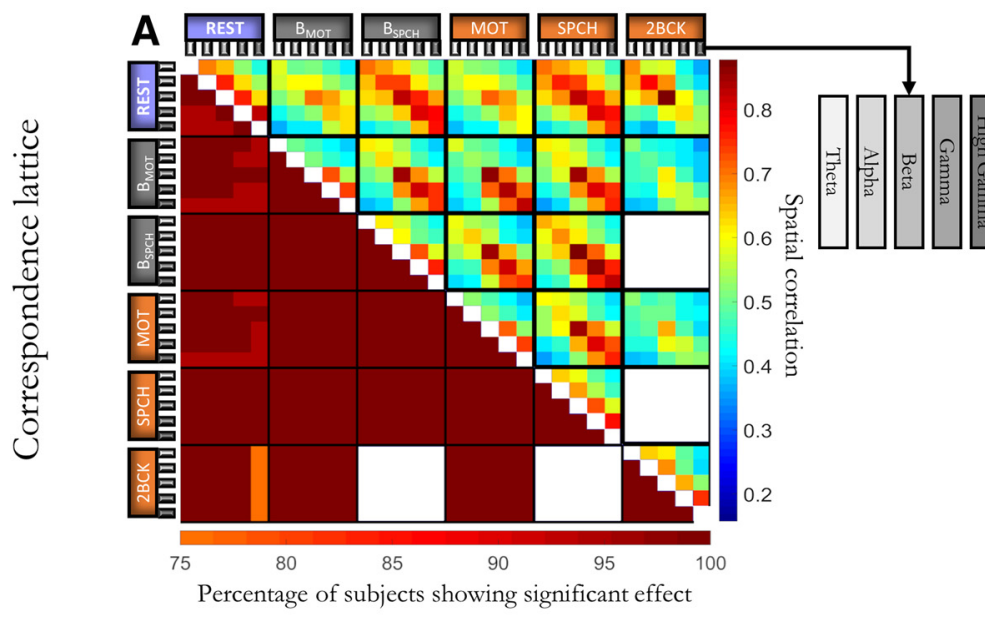

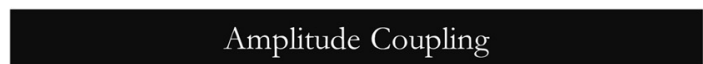

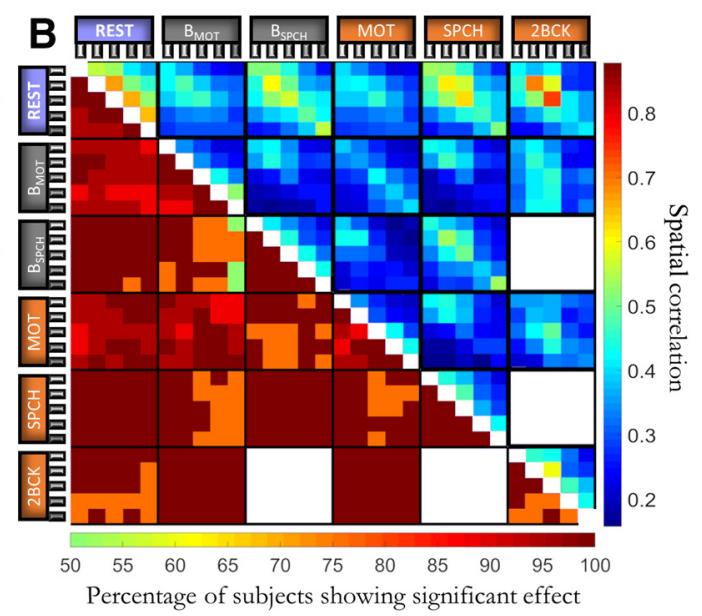

D

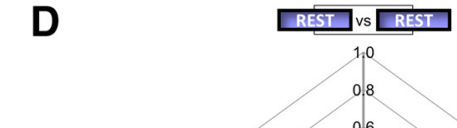

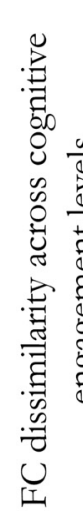

C

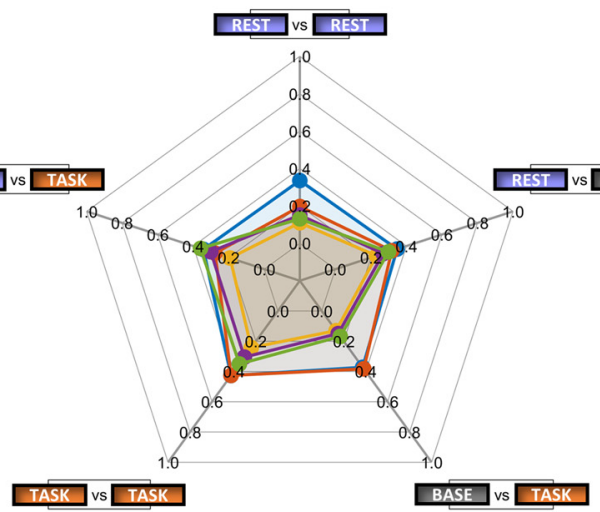

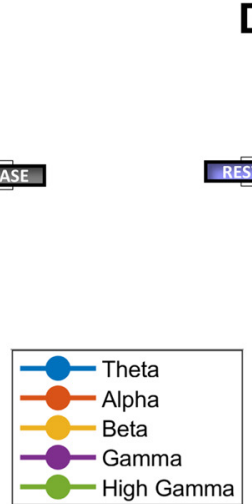

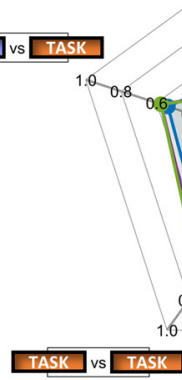

- High Gamm

Figure 6. Spatial correlation between $\mathrm{FC}$ matrices from all possible pairs of cognitive states and frequency bands for phase coupling and amplitude coupling. $\boldsymbol{A}, \boldsymbol{B}$, In the correspondence lat-

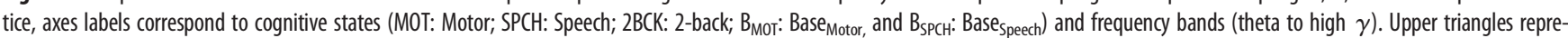
sent correlation values averaged across all subjects, with effect size represented by color (vertical color bar). For each subject, a set of 500 surrogate data were generated to test the significance of the correlation values. The number of subjects showing significance in each bin of the correspondence lattice is presented in the lower triangles (horizontal color bar). $\boldsymbol{A}, \boldsymbol{B}$, Lower triangles conform with Hypothesis IV in Figure 1. C, D, The radar plots show the magnitude of FC dissimilarity $(1-r)$ across all levels of cognitive engagement. Cognitive engagement is conceptualized to gradually increase from resting state (Rest) to intertrial baseline periods during paradigms (Base, averaged over Base Motor $_{\text {and Base }}$ speech), to active processing (Task, averaged over Motor, Speech, and 2-back). Radar plots in different colors correspond to different frequency bands. The comparable magnitude of dissimilarity across all pairs of cognitive engagement levels speaks to the presence of an intrinsic spatial organization for both phase and amplitude coupling.

cognitive states exceeded chance level for all individual subjects and frequency bands with only 2 exceptions of 125 cross-state cases shown in Figure $3(q<0.05$; BenjaminiHochberg corrected for subjects $\times$ frequencies $\times$ cognitive state pairs). This outcome indicates the presence of an intrinsic FC organization of phase coupling in all frequency bands. Results for amplitude coupling were comparable $(r=0.45 \pm$ 0.22 averaged across subjects, frequency bands, and RestMotor, Rest-Speech, and Rest-2-back condition pairs).

Cross-state versus within-state spatial correspondence

Spatial similarity of FC patterns was also assessed within a single cognitive state for comparison. The relatively long duration of resting-state recordings allowed comparing time-averaged FC matrices from two equal halves of the run (Fig. 3, purple data points). Overall, spatial similarity within resting state was either equivalent or only slightly stronger compared with the similarity across cognitive states. Specifically, the average reduction of the $r$ values over all cross-state conditions and frequency bands was $11 \pm 12 \%$ (mean $\pm \mathrm{SD}$ ). Therefore, divergence of spatial organization of FC across different cognitive states is comparably small.
Amplitude coupling showed comparable, albeit slightly larger, reduction values (mean \pm SD: $0.17 \pm 0.16$ ).

\section{Intrinsic FC organization shared across frequency bands}

Above, we showed the presence of an intrinsic FC organization that is stable across cognitive states within each of the canonical frequency bands (Fig. 3). Is a unifying state-invariant FC organization shared across frequency bands, or does each specific frequency have its own unique spatial organization? Here, we assess how the state-invariant FC organization of each frequency band spatially correlates with that of other bands. To this end, for each subject and frequency band, we calculated the geometric mean of the FC matrices from all six cognitive states as the representative intrinsic organization of that frequency band (Fig. $4 A, B$ ). The geometric mean is often used to find the central tendency for different items, emphasizing consistency. In other words, if an electrode pair has a small FC value in even a single cognitive state, that pair obtains a small value in the intrinsic organization matrix since it is not consistent over all cognitive states.

For each subject, we assessed spatial correlations across pairs of representative (mean) intrinsic FC. For each pair of 


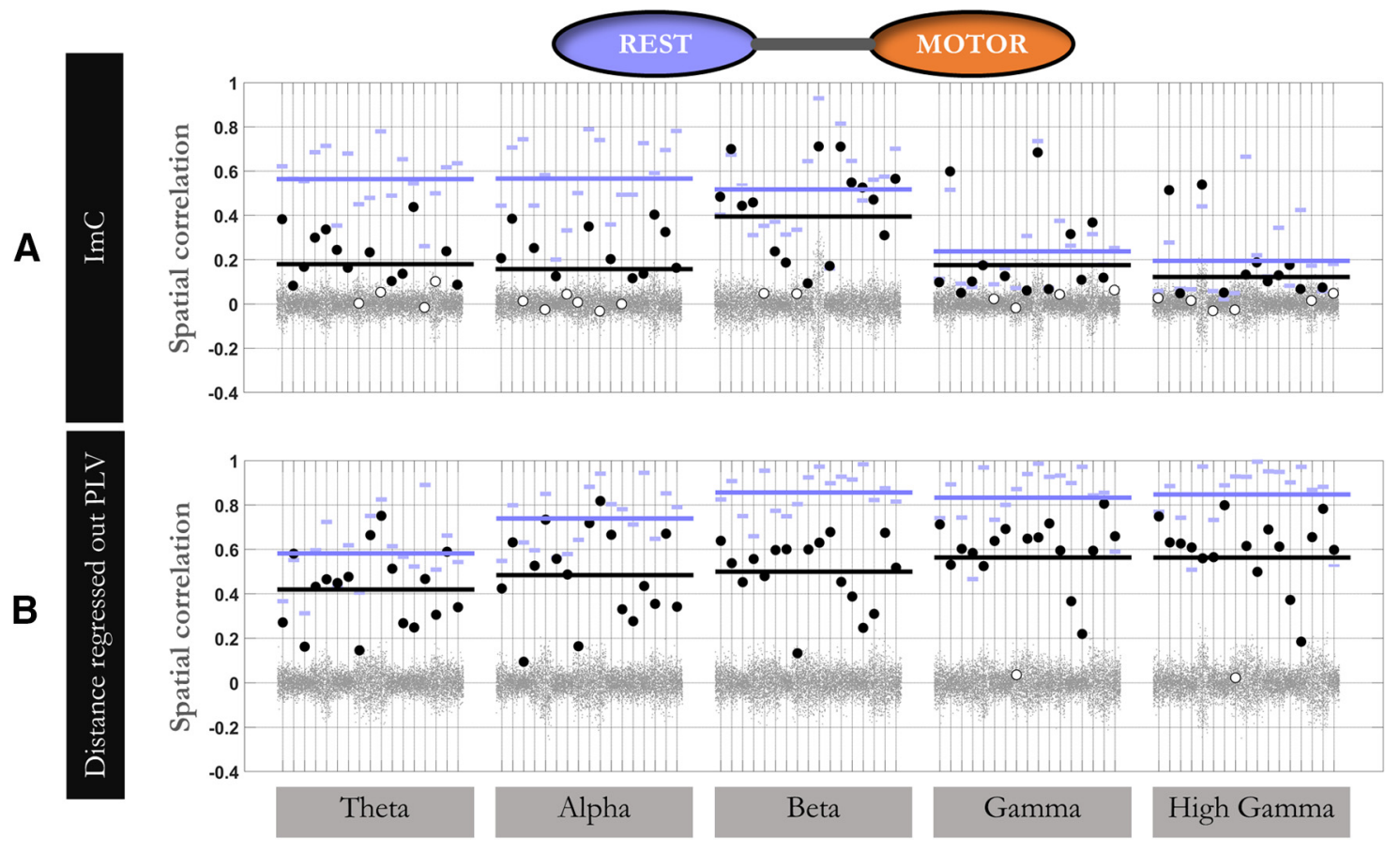

Figure 7. Addressing potential contribution of volume conduction to the cross-state spatial correlation of FC. Rest-to-Motor cross-state FC correlations based on $(\boldsymbol{A})$ ImC measure and $(\boldsymbol{B})$ PLV measure after regressing out electrode distance. Each subplot is organized according to configurations of Figure 3. A large proportion of data shows the presence of cross-state FC correlations, even after removing possible volume conduction effects, using the two different approaches. This observation suggests that our major findings are not heavily driven by volume conduction.

frequencies, we statistically tested the significance of FC similarity using the spatial permutation method described above (500 permutations). At the group level, we found significantly higher cross-frequency similarity of FC organization compared with null data (pairwise $t$ test against the mean correlation from permutations; all $\left.t_{(17)}>9.37, p<2 \mathrm{e}-8\right)$. At the individual level, the cross-frequency spatial similarity exceeded chance level of the null model for all frequency pairs and all subjects $(q<0.05$; FDR-corrected for $18 * C\left(\begin{array}{l}5 \\ 2\end{array}\right)=180$ cases of subjects $\times$ frequency pairs). Figure $4 C$ shows the spatial correlation values averaged over subjects (upper triangle) as well as the number of subjects showing a significant effect (lower triangle). The strong spatial relationship ( $r=0.69-0.88)$ across all frequency pairs demonstrates that FC is governed by a universal spatial organization largely shared across frequency bands. Equivalent results from amplitude coupling were observed $(r=0.45-0.61)$.

\section{Temporal dynamics dissociating broadband versus band- limited FC}

Our observation that the intrinsic FC organization is highly similar across frequency bands may be explained by one of two alternative scenarios. Either temporally independent coupling processes in multiple frequency bands indeed share a unifying spatial organization, or a single broadband coupling process (e.g., coupled bursts with sharp on/offset) underlies FC in all frequencies. In the latter case, FC would be temporally correlated over frequencies. To dissociate between the two scenarios, we inspected temporal correlations between connection-wise FC time courses of different frequency bands within the resting state condition because it provides the most data of all cognitive states (2-3 min). We only focused on those connections of the intrinsic FC organization that were strong in all frequency bands (top 25\% of the representative mean organization illustrated in Fig. $4 B$; mean $\mathrm{FC}=0.60 ; \mathrm{SD}=0.13$; averaged over subjects). This approach ensured that we only included electrode pairs involved in the task-invariant FC organization of all bands. As shown in Figure 5 (top subplots above the diagonal; "real data"), we detected a symmetric histogram of temporal correlation values centered on zero for every subject and pair of frequency bands. The lower subplots below the diagonal of Figure 5 show an equivalent temporal correlation analysis for surrogate data generated by temporally permuting phases of one of the FC dynamics in Fourier space (500 permutations). The distribution of temporal correlations was highly similar to the corresponding histogram of the real data. Again, similar results were obtained for amplitude coupling.

To test for an overall tendency toward positive or negative correlations between FC temporal dynamics of each frequency pair, we compared mean, SD, and skewness between histograms of the real and null data. The (grand average) difference in the three measures between histograms of the real and null data (averaged across repetitions) were $0.01,0.01$, and $0.02( \pm \mathrm{SD}=$ $0.03,0.01$, and 0.13 ), respectively. All differences were negligible in size, suggesting the absence of a broadband process. Moreover, a large proportion of all cases (all subjects, pairs of frequency bands, and the three histogram measures) did not pass the significance test when compared with surrogate data $(73,68$, and $95 \%$ of all cases for mean, SD, and skewness differences, respectively; $q<0.05)$. This observation implies that FC fluctuations in different frequency bands do not temporally coincide (in a linear sense) more frequently than expected by chance. We conclude that the observed spatial concordance of FC organization across frequencies is not generated by a single broadband phenomenon, but rather reflects multiple frequency-specific coupling processes unfolding within the same universal spatial organization. 


\section{Stability of spatial organization across phase and amplitude coupling}

In previous sections, we separately tested the stability of FC organization across cognitive states and frequency bands. Next, to test the four scenarios presented in Figure 1, we assessed the stability of FC organization across all possible pairs of cognitive states and frequency bands. We further extended this comprehensive approach to a comparison across different modes of connectivity (i.e., phase and amplitude coupling).

\section{Spatial organization of phase coupling}

We estimated spatial correlation for all possible pairs of FC matrices across cognitive states and frequencies for each subject separately, resulting in a correspondence lattice (Fig. 6A). For visualization purposes, the upper triangle of the correspondence lattice in Figure $6 \mathrm{~A}$ shows the correspondence lattice averaged over all subjects $(r=0.57 \pm 0.18$, including cross-frequency comparisons; $r=0.69 \pm 0.18$ for within-frequency comparisons only). Using the same spatial permutation test that was used for previous sections, we tested the significance of each bin of the correspondence lattice for each subject. The lower triangle of Figure $6 \mathrm{~A}$ reports the proportion of subjects passing significance threshold for each comparison $(R=500 ; q<0.05$; BenjaminiHochberg FDR-corrected for all cases of subjects $\times$ pairs of cognitive state $\times$ frequency bands). We observed evidence for spatial similarity across all pairs of FC matrices in overall $99.3 \%$ of the single-subject comparisons, strongly supporting Hypothesis IV (compare Fig. 1). This outcome shows that the spatial FC organization is to a large degree consistent across all cognitive states and frequency bands.

We further summarized information from the correspondence lattice according to cognitive engagement levels (Fig. 6C). These levels are conceptualized to gradually increase from taskfree resting state (Rest), to prestimulus baseline involving task-set

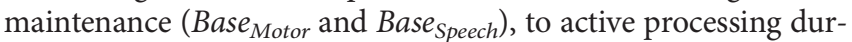
ing stimulation and motor output (Motor, Speech, and 2-back). We focused on the amount of FC dissimilarity $(1-r)$ across pairs of cognitive engagement levels to emphasize the change in FC organization. In all frequency bands, the radar plots approached a circle, showing that the dissimilarity of FC was of comparable magnitude across all level pairs. In other words, more distant pairs of engagement levels, most notably base-task and task-task contrasts as commonly applied in electrophysiological studies, were no more dissimilar than the closer rest-rest pair.

\section{Spatial organization of amplitude coupling}

Amplitude coupling is a distinct mode of oscillation-based FC beyond phase, likewise subserving long-range neural communication and cognitive processes (Engel et al., 2013). We have previously hypothesized that FC organization in both modes entails an intrinsic, task-independent component (Mostame and Sadaghiani, 2020). Therefore, we asked whether the same observation (i.e., consistency across cognitive states and frequency bands) also holds true for amplitude coupling. We extracted FC matrices of all cognitive states and frequency bands using amplitude coupling as connectivity measure. Figure $6 B$ visualizes the outcome for amplitude coupling (equivalent to Fig. $6 \mathrm{~A}$ for phase coupling). Spatial correlation values were moderate to strong (average of upper triangle of Fig. $6 B, r=0.42 \pm 0.21$ ). Importantly, as shown in the lower triangle of Figure $6 B$, spatial correlation of FC matrices across all cognitive states and frequencies was significant in overall $94.7 \%$ of the comparisons $(q<0.05$;
Benjamini-Hochberg FDR-corrected for subjects $\times$ pairs of cognitive state $\times$ frequency bands), in line with Hypothesis IV in Figure 1. In the radar plots for amplitude coupling (Fig. 6D), FC dissimilarity between more distant pairs of cognitive engagement levels were only marginally larger in magnitude compared with the rest-rest pair. We conclude that the intrinsic architecture is also consistent across cognitive states and frequency bands for amplitude coupling.

\section{Comparing spatial organization between phase and amplitude coupling}

We observed differences between phase and amplitude coupling. While spatial correlation values for both were significant compared with their respective null models, values were overall slightly higher for phase coupling (Fig. 6A) than for amplitude coupling (Fig. 6B). Further, as the frequency increased, withinfrequency cross-state correlation values increased for phase coupling (Fig. $6 A, C$, diagonals of small squares), while they slightly decreased for amplitude coupling (Fig. 6B,D). This means that task-specific FC changes in phase coupling are more pronounced in lower frequencies (mostly in alpha band), resulting in lower cross-state correlations. By contrast, such task-specific changes are generally stronger in higher frequencies for amplitude coupling. Collectively, these observations suggest the presence of an intrinsic architecture in both phase and amplitude coupling, but with nuanced differences.

\section{Source leakage contributions}

Compared with EEG and MEG, ECoG recordings are much less likely to suffer from volume conduction, especially at the scale of $\sim>1 \mathrm{~cm}$ interelectrode distance (Dubey and Ray, 2019). Nevertheless, to ascertain that FC stability across cognitive states is largely independent of volume conduction, we replicated our results following suppression of zero-lag connectivity (since electricity spreads quasi-instantaneously) and distance dependence of connectivity (since volume conduction is dependent on physical distance). We used the Rest-to-Motor comparison because of its large number of subjects (Fig. 7).

FC measures suppressing zero-lag connectivity (ImC measure) We found that significant Rest-to-Motor cross-state FC correspondence persisted in the majority of bands and subjects (63 of 85 cases; with a minimum of 11 of 17 subjects in each band), even after reducing the data to nonzero-lag connectivity (Fig. 7A; FDR-corrected at $q<0.05)$. Despite a reduction in effect size likely because of concomitant removal of veridical zero-lag connectivity, the persistence of effects implies that cross-state FC correspondence is not primarily explained by volume conduction effects. Overall, relatively low within-Rest correlation values of FC matrices (Fig. $7 A$, long purple lines; $r=\sim 0.4$ ) speak to low sensitivity of the ImC and the underestimation of the cross-state correlations.

\section{Regressing out distance dependencies from FC}

In 83 of 85 subject $\times$ frequency cases, spatial organization of FC remained significantly correlated between Rest and Motor conditions after removing distance dependencies (Fig. $7 B$ ). The large proportion of significant effects, even after removal of a substantial part of connectivity, indicates that our major findings are not primarily driven by distance dependencies. This persistence suggests that volume conduction contributes no more than a small proportion of the observed consistency of FC organization across cognitive states and frequency bands. 


\section{Discussion}

The present study sought to answer whether fast oscillatory coupling was sensitive to cognitive context, or, conversely, associated with a single, state-invariant spatial organization across cognitive domains. We used ECoG signals of presurgical patients during rest, prestimulus intervals corresponding to maintenance of taskset and attention, and active processing. Across all subjects and all canonical frequency bands, oscillation-based FC spatial organization changed only slightly in response to cognitive state. Moreover, the observed spatial organization was largely similar across all frequency bands. Despite this between-frequency spatial similarity, temporally independent dynamics were detected across frequency bands speaking against a broadband phenomenon. Together, we observed cognitive state invariance and frequency invariance of the distributed FC spatial organization on the one hand, and frequency-specific FC dynamics at the connection level on the other.

To illustrate the concurrent presence of these properties, we consider the analogy of vehicle traffic. In this analogy, the distributed spatial FC organization corresponds to the pattern of roads, and dynamic FC corresponds to traffic unfolding on the roads. Our result of state invariance of the spatial organization can be conceptualized as the stability of the roads' network pattern irrespective of the ambient conditions, such as weather. Illustrating the frequency invariance of FC spatial organization, different frequency bands correspond to individual lanes within the same road layout. The absence of linear dependence of FC dynamics across bands then corresponds to cars travelling in a temporally largely independent manner on the distinct lanes of the same roads. This analogy highlights how our results extend the neurobiological understanding of functional networks through the concurrent consideration of distributed spatial organization and temporal dynamics of FC.

\section{Stability of oscillation-based FC across cognitive states}

Spatial stability of fast oscillation-based FC over cognitive states is in strong agreement with prior observations in fMRI-based FC at infra-slow temporal scales. Several neuroimaging studies have shown that the spatial organization of FC remains largely stable across cognitive states (Cole et al., 2014; Krienen et al., 2014; Gratton et al., 2018). However, the vastly different temporal characteristics of fMRI and electrophysiological measures emphasize divergent types of neural processes (Hari and Parkkonen, 2015; Hermes et al., 2017, 2019). fMRI-based FC represents coupling of infra-slow and aperiodic fluctuations of activation amplitude. In contrast, coupling of oscillatory neural signals, especially phase coupling, represent FC mechanisms based on rapid rhythmicity.

The strong spatial stability in our study $(r=0.69$; mean over all cross-state comparisons of phase coupling within frequencies) is especially surprising in light of the role of neural oscillations in rapid cognitive processes. This state invariance of FC organization is missed in common electrophysiological task-based studies, as such studies typically normalize active trial processing to a pretrial baseline or contrast task conditions in individual connections without considering the distributed spatial pattern of FC.

However, the observed stability leaves room for subtle or spatially confined task-specific FC changes. It is important to consider the interplay between space and time in FC dynamics. Our observation of spatial stability of FC in various cognitive contexts over the full trial duration does not preclude divergent temporal dynamics in different tasks (see Discussion of time-varying dynamics below). Further, spatial correspondence of FC across states was high but not perfect, suggesting the presence of task-specific adjustments. This latter observation is in line with small, but task-specific, changes in fMRI-based FC during particular tasks (e.g., J. R. Cohen and D'Esposito, 2016). Thus, the observed spatial stability is compatible with additional minor but functionally important and taskspecific changes in FC patterns.

\section{Stability of oscillation-based FC across frequency bands}

The cognitive state invariance of oscillation-based FC organization was observed in all canonical frequency bands from theta to high $\gamma$, suggesting that this spatial organization is conserved across frequencies. Direct pairwise comparisons of frequencyspecific intrinsic FC across frequency bands revealed highly similar FC organization across all bands $(r=0.69-0.88)$. This finding is surprising, given that frequency-specific oscillation-based FC has been linked to different cognitive operations, each associated with different sets of brain areas (S. Palva and Palva, 2018). For instance, theta, $\alpha$, and $\gamma$ band oscillations (both in terms of local power and cross-region coupling) are thought to reflect navigation and memory encoding/retrieval (Backus et al., 2016), attentional processes (Sadaghiani and Kleinschmidt, 2016), and local representation of item content (Rohenkohl et al., 2018), respectively. These observations would suggest a frequency-specific rather than a frequency-stable spatial organization of FC (Sadaghiani and Wirsich, 2020).

However, computer modeling studies have shown that spatial organization of static FC in various frequency bands can be largely predicted by the structural connectivity (Cabral et al., 2014; Hansen et al., 2015; Schirner et al., 2018), suggesting some degree of frequency invariance in the organization of FC. For example, Cabral et al. (2014) reported strong correlation between empirical FC of MEG data and time series generated from theoretical computer models informed by structural connectivity. Importantly, this observation concurrently held true for multiple oscillation frequency bands, suggesting that oscillation-based FC entails a frequency-independent component. Our observation of relatively strong frequency invariance agrees with this viewpoint, while also permitting considerably smaller frequency-specific connectivity patterns.

\section{Temporally independent frequency-specific FC dynamics}

Our analysis of FC time-varying dynamics in each frequency band suggests that frequency invariance likely reflects multiple frequency-specific coupling processes that enact a shared spatial organization, rather than a single broadband process. This finding may explain how frequency-specific task-evoked FC changes can coexist with a frequency-invariant spatial organization. Although temporally independent FC dynamics in different frequency bands are consistent with temporally distinct task-evoked changes in each band, these results are dependent on the time frame considered. For instance, when assessing these connection-wise dynamics over more extensive observations (i.e., longer time period), the relatively unitary state- and frequency-invariant spatial organization of FC emerges at the large-scale connectivity level. Our finding is consistent with long-term EEG and ECoG recordings that report the emergence of spatial stability only at periods $>100$ s (Kramer et al., 2011; Chu et al., 2012). This observation reconciles the complimentary presence of cognitive state invariance and frequency invariance of FC spatial organization on the one hand and its state-responsive and frequency-specific short-term dynamics on the other. 


\section{Phase versus amplitude coupling}

Beyond phase coupling, this study explored the correspondence of spatial FC across cognitive states and frequency bands in the other major mode of oscillation-based connectivity: amplitude coupling. While relative stability of FC organization was observed for both coupling modes, the cross-state FC dissimilarity for amplitude coupling was consistently larger than for phase coupling in all bands (Fig. 6C), slightly exceeding dissimilarity within rest (Fig. 6D). This finding may suggest that amplitude coupling is modulated across cognitive contexts either to a larger degree or across more connections than phase coupling.

Further, we observed that task responsiveness of phase and amplitude coupling had a different profile over frequency bands (Fig. 6C,D). The observed profiles suggest that task-related changes in phase coupling occur more readily in slower frequencies, whereas task-related amplitude coupling enacts the higher frequencies more strongly. The dissociable task-related spatial reorganization emphasizes the distinctness of task-related phase and amplitude coupling (Mostame and Sadaghiani, 2020).

\section{Source leakage contributions}

Could the observed state and frequency invariance be caused by volume conduction present in the data regardless of the cognitive state or frequency band? Although ECoG is considerably less affected by volume conduction than scalp EEG, we addressed this concern after removing zero-lag FC and distance dependence of FC. We detected a moderate reduction of the effect size of the spatial correspondence, especially when suppressing zero-lag FC. Unfortunately, the conservative approach of suppressing zero-lag FC comes at the cost of removing real zero-lag connectivity whose existence (e.g., Gray et al., 1989; Roelfsema et al., 1997; Rodriguez et al., 1999) and contribution to the whole-brain connectome (e.g., Finger et al., 2016) are supported empirically and theoretically (Viriyopase et al., 2012). Importantly, however, a large proportion of the data still reflected the state-invariant FC organization, indicating that volume conduction is not a primary driver of our effects. These observations emphasize the advantage of ECoG over noninvasive neurophysiological signals for investigating FC organization under minimal volume conduction effects.

\section{Limitations}

While ECoG provides a unique window into direct intracranial recordings of the human brain, it suffers from limited electrode coverage. Thus, the observed FC organization could not be directly related to previously reported whole-brain FC networks. Importantly however, the core question regarding spatial stability of FC across cognitive states and frequency bands was not contingent on knowing the correspondence to MRI-based networks. Moreover, the variability of electrode coverage across subjects, while it prevents comparisons across subjects, suggests that our observations are robust over different sets of brain areas.

Another limitation of ECoG is that, because of its invasive nature, it is only available in patients with a history of epilepsy. In particular, electrode coverage usually includes affected brain areas to serve clinical purposes. However, data did not include electrodes and time periods with excessive interictal activity. Thus, we believe that the high signal-to-noise ratio of ECoG and its relative insensitivity to volume conduction far outweighs this potential limitation for the purposes of studying oscillationbased FC. Thereby our study adds to the wealth of prior ECoG studies successfully informing about normal brain processes (Parvizi and Kastner, 2018) and FC in particular (e.g., Kucyi et al., 2018; Betzel et al., 2019).
Another limitation ensuing from the rare opportunity of intracranial human recordings is the limited sample size. Crossstate investigations require patients with data from at least two task conditions. The overlap of Motor and Rest provided a sample size of 17 patients, which is relatively large in the context of ECoG literature. However, other tasks that we included in support of a broad representation of cognitive states were available in a subset of patients only. Nevertheless, the extraordinary signal-to-noise ratio of intracranial recordings permits assessment of effects in individual subjects. Accordingly, the core conclusions of state and frequency invariance were established using both group-level statistics as well as single-subject tests using subject-specific null models. The observation of all core findings in the vast majority of individual comparisons (with rigorous multiple comparisons corrections) provides strong quantitative support for the robustness of our results.

In conclusion, our findings suggest that spatial organization of oscillation-based FC is shared across frequency bands and is stable across a variety of tasks and rest. Invariance of oscillation-based FC across cognitive states agrees with parallel observations of such invariance in the correlation structure of the aperiodic fMRI BOLD signal. This convergence suggests a universal phenomenon from the millisecond timescale of electrophysiology to the infra-slow range of fMRI. These observations speak to conceptual frameworks of oscillation-based FC incorporating a largely state- and frequencyinvariant spatial organization beyond state-responsive and frequency-specific short-term dynamics of FC (Sadaghiani and Wirsich, 2020).

Such conceptual considerations also have important practical implications for calculating and interpreting task-based electrophysiological connectivity. This is especially important because commonly used nonparametric statistical tests (phase permutation, Bootstrap resampling, etc.) differ in the adequacy with which their assumed null distribution captures task-independent connectivity already present in the prestimulus baseline (Moharramipour et al., 2018; Mostame et al., 2019). Intrinsic connectivity can be taken into account by either demeaning connectivity at each connection with respect to its corresponding baseline connectivity before statistical testing, or by using random sampling approaches that include baseline data (Mostame et al., 2019).

The strong similarity of spatial FC organization across mental states also motivates dedicated studies to characterize the subtle but functionally meaningful state-dependent modulation of this distributed organization. Given sufficient spatial localizability (in either distributed intracranial data or whole-brain source-localized scalp data), comparisons between task conditions can be performed using network-based statistical approaches rather than studying individual connections (e.g., Zalesky et al., 2010). In summary, concurrently considering the distributed spatial organization and temporal dynamics of FC in future task-based studies will be important to advance the neurobiological understanding of functional networks.

\section{References}

Backus AR, Schoffelen JM, Szebényi S, Hanslmayr S, Doeller CF (2016) Hippocampal-prefrontal theta oscillations support memory integration. Curr Biol 26:450-457.

Beckmann CF, DeLuca M, Devlin JT, Smith SM (2005) Investigations into resting-state connectivity using independent component analysis. Philos Trans R Soc Lond B Biol Sci 360:1001-1013.

Berger H (1930) Ueber Das Elektrenkephalogramm Des Menschen. [Electrocephalography in Man.]. J Psychologie Neurologie 40:160-179. 
Betzel RF, Medaglia JD, Kahn AE, Soffer J, Schonhaut DR, Bassett DS (2019) Structural, geometric and genetic factors predict interregional brain connectivity patterns probed by electrocorticography. Nat Biomed Eng 1:902-916.

Brookes MJ, Woolrich M, Luckhoo H, Price D, Hale JR, Stephenson MC, Barnes GR, Smith SM, Morris PG (2011) Investigating the electrophysiological basis of resting state networks using magnetoencephalography. Proc Natl Acad Sci USA 108:16783-16788.

Cabral J, Luckhoo H, Woolrich M, Joensson M, Mohseni H, Baker A, Kringelbach ML, Deco G (2014) Exploring mechanisms of spontaneous functional connectivity in MEG: how delayed network interactions lead to structured amplitude envelopes of band-pass filtered oscillations. Neuroimage 90:423-435.

Chu CJ, Kramer MA, Pathmanathan J, Bianchi MT, Westover MB, Wizon L, Cash SS (2012) Emergence of stable functional networks in long-term human electroencephalography. J Neurosci 32:2703-2713.

Cohen JR, D'Esposito M (2016) The segregation and integration of distinct brain networks and their relationship to cognition. J Neurosci 36:1208312094.

Cohen MX (2015) Effects of time lag and frequency matching on phasebased connectivity. J Neurosci Methods 250:137-146.

Colclough GL, Woolrich MW, Tewarie PK, Brookes MJ, Quinn AJ, Smith SM (2016) How reliable are MEG resting-state connectivity metrics? Neuroimage 138:284-293.

Cole MW, Bassett DS, Power JD, Braver TS, Petersen SE (2014) Intrinsic and task-evoked network architectures of the human brain. Neuron 83:238251.

Deligianni F, Centeno M, Carmichael DW, Clayden JD (2014) Relating resting-state FMRI and EEG whole-brain connectomes across frequency bands. Front Neurosci 8:258.

de Medeiros Kanda PA, Anghinah R, Smidth MT, Silva JM (2009) The clinical use of quantitative EEG in cognitive disorders. Dement Neuropsychol 3:195-203.

Dubey A, Ray S (2019) Cortical electrocorticogram (ECoG) is a local signal. J Neurosci 39:4299-4311.

Engel AK, Gerloff C, Hilgetag CC, Nolte G (2013) Intrinsic coupling modes: multiscale interactions in ongoing brain activity. Neuron 80:867-886.

Fell J, Axmacher N (2011) The role of phase synchronization in memory processes. Nat Rev Neurosci 12:105-118.

Finger H, Bönstrup M, Cheng B, Messé A, Hilgetag C, Thomalla G, Gerloff C, König P (2016) Modeling of large-scale functional brain networks based on structural connectivity from DTI: comparison with EEG derived phase coupling networks and evaluation of alternative methods along the modeling path. PLoS Comput Biol 12:e1005025.

Fox KC, Foster BL, Kucyi A, Daitch AL, Parvizi J (2018) Intracranial electrophysiology of the human default network. Trends Cogn Sci 22:307-324.

Fox MD, Raichle ME (2007) Spontaneous fluctuations in brain activity observed with functional magnetic resonance imaging. Nat Rev Neurosci 8:700-711.

Gratton C, Laumann TO, Nielsen AN, Greene DJ, Gordon EM, Gilmore AW, Nelson SM, Coalson RS, Snyder AZ, Schlaggar BL, Dosenbach NU, Petersen SE (2018) Functional brain networks are dominated by stable group and individual factors, not cognitive or daily variation. Neuron 98:439-452.e5.

Gray CM, König P, Engel AK, Singer W (1989) Oscillatory responses in cat visual cortex exhibit inter-columnar synchronization which reflects global stimulus properties. Nature 338:334-337.

Gruber MJ, Hsieh LT, Staresina BP, Elger CE, Fell J, Axmacher N, Ranganath C (2018) Theta phase synchronization between the human hippocampus and prefrontal cortex increases during encoding of unexpected information: a case study. J Cogn Neurosci 30:1646-1656.

Hansen EC, Battaglia D, Spiegler A, Deco G, Jirsa VK (2015) Functional connectivity dynamics: modeling the switching behavior of the resting state. Neuroimage 105:525-535.

Hari R, Parkkonen L (2015) The brain timewise: how timing shapes and supports brain function. Philos Trans R Soc Lond B Biol Sci 370:20140170.

Hearne LJ, Cocchi L, Zalesky A, Mattingley JB (2017) Reconfiguration of brain network architectures between resting-state and complexitydependent cognitive reasoning. J Neurosci 37:8399-8411.

Hermes D, Nguyen M, Winawer J (2017) Neuronal synchrony and the relation between the blood-oxygen-level dependent response and the local field potential. PLoS Biol 15:e2001461.
Hermes D, Petridou N, Kay KN, Winawer J (2019) An image-computable model for the stimulus selectivity of gamma oscillations. eLife 8:e47035.

Hillebrand A, Barnes GR, Bosboom JL, Berendse HW, Stam CJ (2012) Frequency-dependent functional connectivity within resting-state networks: an atlas-based MEG beamformer solution. Neuroimage 59:39093921.

Hipp JF, Siegel M (2015) BOLD fMRI correlation reflects frequency-specific neuronal correlation. Curr Biol 25:1368-1374.

Jensen O, Gips B, Bergmann TO, Bonnefond M (2014) Temporal coding organized by coupled alpha and gamma oscillations prioritize visual processing. Trends Neurosci 37:357-369.

Khanna P, Carmena JM (2015) Neural oscillations: beta band activity across motor networks. Curr Opin Neurobiol 32:60-67.

Kramer MA, Eden UT, Lepage KQ, Kolaczyk ED, Bianchi MT, Cash SS (2011) Emergence of persistent networks in long-term intracranial EEG recordings. J Neurosci 31:15757-15767.

Krienen FM, Yeo BT, Buckner RL (2014) Reconfigurable task-dependent functional coupling modes cluster around a core functional architecture. Philos Trans R Soc Lond B Biol Sci 369:20130526.

Kucyi A, Schrouff J, Bickel S, Foster BL, Shine JM, Parvizi J (2018) Intracranial electrophysiology reveals reproducible intrinsic functional connectivity within human brain networks. J Neurosci 38:4230-4242.

Lachaux JP, Rodriguez E, Martinerie J, Varela FJ (1999) Measuring phase synchrony in brain signals. Hum Brain Mapp 8:194-208.

Miller KJ (2019) A library of human electrocorticographic data and analyses. Nat Hum Behav 3:1225-1235.

Miller KJ, Leuthardt EC, Schalk G, Rao RP, Anderson NR, Moran DW, Miller JW, Ojemann JG (2007) Spectral changes in cortical surface potentials during motor movement. J Neurosci 27:2424-2432.

Miller KJ, Sorensen LB, Ojemann JG, den Nijs M (2009) Power-law scaling in the brain surface electric potential. PLoS Comput Biol 5:e1000609.

Miller KJ, Hermes D, Honey CJ, Hebb AO, Ramsey NF, Knight RT, Ojemann JG, Fetz EE (2012) Human motor cortical activity is selectively phase-entrained on underlying rhythms. PLoS Comput Biol 8:e1002655.

Miller KJ, Taylor JA, Adam OH, Jeffrey GO (2011) Rapid Online Language Mapping with Electrocorticography. Journal of Neurosurgery. Pediatrics 7:482-490.

Moharramipour A, Mostame P, Hossein-Zadeh G-A, Wheless JW, BabajaniFeremi A (2018) Comparison of Statistical Tests in Effective Connectivity Analysis of ECoG Data. Journal of Neuroscience Methods 308:317-329.

Mostame P, Sadaghiani S (2020) Phase and amplitude coupling are tied by an intrinsic spatial organization but show divergent stimulus-related changes. Neuroimage 219:117051.

Mostame P, Moharramipour A, Hossein-Zadeh GA, Babajani-Feremi A (2019) Statistical significance assessment of phase synchrony in the presence of background couplings: an ECoG study. Brain Topogr 32:882896.

Nentwich M, Ai L, Madsen J, Telesford QK, Haufe S, Milham MP, Parra LC (2020) Functional connectivity of EEG is subject-specific, associated with phenotype, and different from fMRI. Neuroimage 218:117001.

Nolte G, Bai O, Wheaton L, Mari Z, Vorbach S, Hallett M (2004) Identifying true brain interaction from EEG data using the imaginary part of coherency. Clin Neurophysiol 115:2292-2307.

Oostenveld R, Fries P, Maris E, Schoffelen JM (2011) FieldTrip: open source software for advanced analysis of MEG, EEG, and invasive electrophysiological data. Intell Neurosci 2011:1-9.

Palva JM, Wang SH, Palva S, Zhigalov A, Monto S, Brookes MJ, Schoffelen JM, Jerbi K (2018) Ghost interactions in MEG/EEG source space: a note of caution on inter-areal coupling measures. Neuroimage 173:632-643.

Palva S, Palva JM (2007) New vistas for $\alpha$-frequency band oscillations. Trends Neurosci 30:150-158.

Palva S, Palva JM (2018) Roles of brain criticality and multiscale oscillations in temporal predictions for sensorimotor processing. Trends Neurosci 41:729-743.

Parvizi J, Kastner S (2018) Promises and limitations of human intracranial electroencephalography. Nat Neurosci 21:474-483.

Petersen SE, Sporns O (2015) Brain networks and cognitive architectures. Neuron 88:207-219.

Prichard D, Theiler J (1994) Generating surrogate data for time series with several simultaneously measured variables. Phys Rev Lett 73:951-954.

Rimmele JM, Gross J, Molholm S, Keitel A (2018) Editorial: brain oscillations in human communication. Front Hum Neurosci 12:39. 
Rodriguez E, George N, Lachaux JP, Martinerie J, Renault B, Varela FJ (1999) Perception's shadow: long-distance synchronization of human brain activity. Nature 397:430-433.

Roelfsema PR, Engel AK, König P, Singer W (1997) Visuomotor integration is associated with zero time-lag synchronization among cortical areas. Nature 385:157-161.

Rogers N, Hermiz J, Ganji M, Kaestner E, Kılıç K, Hossain L, Thunemann M, Cleary DR, Carter BS, Barba D, Devor A, Halgren E, Dayeh SA, Gilja V (2019) Correlation structure in micro-ECoG recordings is described by spatially coherent components. PLoS Comput Biol 15:e1006769.

Rohenkohl G, Bosman CA, Fries P (2018) Gamma synchronization between V1 and V4 improves behavioral performance. Neuron 100:953-963.e3.

Rouse AG, Williams JJ, Wheeler JJ, Moran DW (2016) Spatial co-adaptation of cortical control columns in a micro-ECoG brain-computer interface. J Neural Eng 13:056018.

Sadaghiani S, Kleinschmidt A (2013) Functional interactions between intrinsic brain activity and behavior. Neuroimage 80:379-386.

Sadaghiani S, Kleinschmidt A (2016) Brain networks and $\alpha$-oscillations: structural and functional foundations of cognitive control. Trends Cogn Sci 20:805-817.

Sadaghiani S, Wirsich J (2020) Intrinsic connectome organization across temporal scales: new insights from cross-modal approaches. Netw Neurosci 4:1-49.

Sadaghiani S, Scheeringa R, Lehongre K, Morillon B, Giraud AL, D’Esposito M, Kleinschmidt A (2012) Alpha-band phase synchrony is related to activity in the fronto-parietal adaptive control network. J Neurosci 32:14305-14310.

Sadaghiani S, Dombert PL, Løvstad M, Funderud I, Meling TR, Endestad T, Knight RT, Solbakk AK, D’Esposito M (2019) Lesions to the fronto-parietal network impact alpha-band phase synchrony and cognitive control. Cereb Cortex 29:4143-4153.

Schirner M, McIntosh AR, Jirsa V, Deco G, Ritter P (2018) Inferring multiscale neural mechanisms with brain network modelling. eLife 7:e28927.
Schoffelen JM, Gross J (2009) Source connectivity analysis with MEG and EEG. Hum Brain Mapp 30:1857-1865.

Singer W (1999) Neuronal synchrony: a versatile code for the definition of relations? Neuron 24:49-65.

Singer W (2013) Cortical dynamics revisited. Trends Cogn Sci 17:616-626.

Smith SM, Fox PT, Miller KL, Glahn DC, Fox PM, Mackay CE, Filippini N, Watkins KE, Toro R, Laird AR, Beckmann CF (2009) Correspondence of the brain's functional architecture during activation and rest. Proc Natl Acad Sci USA 106:13040-13045.

Sockeel S, Schwartz D, Pélégrini-Issac M, Benali H (2016) Large-scale functional networks identified from resting-state EEG using spatial ICA. PLoS One 11:e0146845.

Tewarie P, Bright MG, Hillebrand A, Robson SE, Gascoyne LE, Morris PG, Meier J, Van Mieghem P, Brookes MJ (2016) Predicting haemodynamic networks using electrophysiology: the role of non-linear and cross-frequency interactions. Neuroimage 130:273-292.

Thompson WH, Fransson P (2015) The frequency dimension of FMRI dynamic connectivity: network connectivity, functional hubs and integration in the resting brain. Neuroimage 121:227-242.

Uhlhaas PJ, Pipa G, Lima B, Melloni L, Neuenschwander S, Nikolić D, Singer W (2009) Neural synchrony in cortical networks: history, concept and current status. Front Integr Neurosci 3:17.

VanRullen R (2016) Perceptual cycles. Trends Cogn Sci 20:723-735.

Varela F, Lachaux JP, Rodriguez E, Martinerie J (2001) The Brainweb: phase synchronization and large-scale integration. Nat Rev Neurosci 2:229239.

Viriyopase A, Bojak I, Zeitler M, Gielen S (2012) When long-range zero-lag synchronization is feasible in cortical networks. Front Comput Neurosci $6: 49$.

Wirsich J, Ridley B, Besson P, Jirsa V, Bénar C, Ranjeva JP, Guye M (2017) Complementary contributions of concurrent EEG and FMRI connectivity for predicting structural connectivity. Neuroimage 161:251-260.

Zalesky A, Alex F, Edward TB (2010) Network-Based Statistic: Identifying Differences in Brain Networks. NeuroImage 53:1197-1207. 